\title{
A worldwide survey of chronic cough: a manifestation of enhanced somatosensory response
}

\author{
Alyn H. Morice ${ }^{1}$, Adam D. Jakes ${ }^{1}$, Shoaib Faruqi', Surinder S. Birring ${ }^{2}$, \\ Lorcan McGarvey ${ }^{3}$, Brendan Canning ${ }^{4}$, Jaclyn A. Smith ${ }^{5}$, Sean M. Parker ${ }^{6}$, \\ Kian Fan Chung ${ }^{7}$, Kefang Lai ${ }^{8}$, Ian D. Pavord ${ }^{9}$, Jan van den Berg ${ }^{10}$, \\ Woo-Jung Song ${ }^{11}$, Eva Millqvist ${ }^{12}$, Michael J. Farrell ${ }^{13}$, Stuart B. Mazzone ${ }^{14}$, \\ Peter Dicpinigaitis ${ }^{15}$ and The Chronic Cough Registry
}

\begin{abstract}
Affiliations: ${ }^{1}$ Respiratory Medicine, Castle Hill Hospital, Centre for Cardiovascular and Metabolic Research, Hull York Medical School, Cottingham, UK. 'Division of Asthma, Allergy and Lung Biology, King's College London, London, UK. ${ }^{3}$ Dept of Medicine, Institute of Clinical Science, The Queen's University of Belfast, Belfast, UK. ${ }^{4}$ Dept of Medicine, Johns Hopkins Asthma and Allergy Center, Baltimore, MD, USA. ${ }^{5}$ Centre for Respiratory and Allergy, University of Manchester, University Hospital of South Manchester, Manchester, UK. ${ }^{6}$ Dept of Respiratory Medicine, North Tyneside General Hospital, Northumbria Healthcare NHSFT, North Shields, UK. ${ }^{7}$ Royal Brompton and Harefield NHS Trust and National Heart and Lung Institute, Imperial College, London, UK. ${ }^{8}$ Dept of Clinical Research, State Key Laboratory of Respiratory Disease, Guangzhou Institute of Respiratory Disease, First Affiliated Hospital of Guangzhou Medical College, Guangzhou, China. ${ }^{9}$ Nuffield Dept of Medicine Research Building, University of Oxford, Oxford, UK. ${ }^{10}$ Isala klinieken, Zwolle, The Netherlands. ${ }^{11}$ Dept of Internal Medicine, Seoul National University College of Medicine, Seoul, South Korea. ${ }^{12}$ Dept of Internal Medicine/Respiratory Medicine and Allergology, University of Gothenburg, Sahlgrenska University Hospital, Gothenburg. Sweden. ${ }^{13}$ Florey Institute of Neuroscience and Mental Health. Parkville, Australia. ${ }^{14}$ School of Biomedical Sciences, University of Queensland, Brisbane, Australia. ${ }^{15}$ Albert Einstein College of Medicine and Montefiore Medical Center, Bronx, NY, USA.
\end{abstract}

Correspondence: Alyn H. Morice, Head of Cardiorespiratory Studies, Hull York Medical School, Castle Hill Hospital, Cottingham, HU16 5JQ, UK. E-mail: A.H.Moricedhull.ac.uk

ABSTRACT Reports from individual centres suggest a preponderance of females with chronic cough. Females also have heightened cough reflex sensitivity. Here we have reviewed the age and sex of unselected referrals to 11 cough clinics. To investigate the cause of any observed sex dimorphism, functional magnetic resonance imaging of putative cough centres was analysed in normal volunteers.

The demographic profile of consecutive patients presenting with chronic cough was evaluated. Cough challenge with capsaicin was undertaken in normal volunteers to construct a concentration-response curve. Subsequent functional magnetic resonance imaging during repeated inhalation of sub-tussive concentrations of capsaicin observed areas of activation within the brain and differences in the sexes identified.

Of the 10032 patients presenting with chronic cough, two-thirds (6591) were female (mean age 55 years). The patient profile was largely uniform across centres. The most common age for presentation was 60-69 years. The maximum tolerable dose of inhaled capsaicin was lower in females; however, a significantly greater activation of the somatosensory cortex was observed.

Patients presenting with chronic cough from diverse racial and geographic backgrounds have a strikingly homogeneous demographic profile, suggesting a distinct clinical entity. The preponderance of females may be explained by sex-related differences in the central processing of cough sensation.

@ERSpublications

Chronic cough in specialist clinics have homogenous demographic profiles, suggesting single clinical entity http://ow.ly/zrNAa

For editorial comments see page 1103.

This article has supplementary material available from erj.ersjournals.com

Received: Dec 132013 | Accepted after revision: April 18 2014 | First published online: Sept 032014

Copyright @ERS 2014 


\section{Introduction}

Cough is the most common complaint leading patients to seek medical attention [1]. While the majority of these consultations reflect acute viral illnesses, a substantial degree of morbidity is associated with chronic cough; arbitrarily defined in the American College of Chest Physicians' (ACCP) guidelines as a cough being the sole or predominant symptom lasting for a minimum of 8 weeks, with no radiographic evidence of lung disease [2]. Chronic cough may be present in conditions such as asthma, pulmonary fibrosis, lung cancer, or chronic obstructive pulmonary disease (COPD). However the majority of patients presenting with chronic cough do not easily fit into these diagnostic labels, and indeed there is frequently no obvious indicators as to the underlying diagnosis. However, patients suffer a marked decrement in quality of life comparable to patients with disseminated lung cancer or end-stage COPD $[3,4]$. This adverse impact on quality of life is under recognised by clinicians, according to the recent European Respiratory Society (ERS) survey [5].

The British Thoracic Society Cough Guideline Group recommended the referral of patients with a difficultto-treat chronic cough to a specialist clinic and the establishment of a directory of such specialist centres [6]. Reports from individual cough clinics have revealed a marked predominance of middle-aged female patients, although there are no international multicentre studies looking into the demographic profile of patients with chronic cough [7]. If these demographic observations are consistent across large and diverse populations then it suggests important sex differences in the neurophysiology of this vital protective reflex. Females are known to have a heightened response to a wide variety of tussive stimuli and this may be reflected in sex-related differences in the central processing of noxious afferent sensation [8].

In the present study we have analysed data on the age and sex of unselected referrals to 11 specialist cough clinics from Europe, North America and Asia. To assess whether any observed sex-related disparity in clinical demographics is mirrored by neuro-anatomical differences in the central-nervous system processing a retrospective review of functional magnetic resonance imaging (fMRI) of putative cough centres was undertaken.

\section{Methods}

In 2013 the members of the International Cough Registry performed a retrospective review of patients attending their specialist cough clinics. Patients with persistent cough, as defined previously and without significant radiological abnormality, were selected. The study was undertaken by examination of the individual clinic databases, extracting data for the age and sex of the patients at presentation. Data was summated to provide the overall profile and individual clinics were compared. Anonymised patient data was collected to assess the demographic profile of patients presenting to cough clinics.

To examine sex-related neuro-anatomical divergence, age and handedness matched male and female healthy volunteers underwent an inhalation cough challenge with capsaicin and a concentration response curve was constructed. The maximum dose of capsaicin that could be inhaled without coughing was subsequently administered to assess brain activations associated with the urge-to-cough as previously described [9-11]. In brief, an $18 \mathrm{~s}$ challenge was repeated at $60 \mathrm{~s}$ intervals for a minimum of eight challenges. Blood oxygen level dependent (BOLD) fMRI data was collected using a Siemens Trio 3T scanner (Siemens Medical Systems, Erlangen, Germany) to quantify the activity of the neural networks subtending cough. Analysis of the brain imaging data was performed using validated protocols as described previously [12]. Parameter estimates showing the magnitude of hemodynamic dependent changes in BOLD signal intensity, associated with capsaicin-evoked activations, were extracted and compared to assess differences in regional response patterns between pairs of male and female participants. Data were obtained from fMRI experiments conducted under ethics approvals granted by the Melbourne Health Human Research Ethics Committee, Australia (HREC2007.012 and HREC2010.085). T-tests were used for statistical comparisons between evoked responses. The study adheres to the STROBE statement (strengthening the reporting of observational studies in epidemiology).

\section{Results}

A total of 10032 patients were seen in the clinics from November 2003 to March 2013. The database consisted of $n=2219$ Hull (UK), $n=1841$ Holland (The Netherlands), $n=1518$ Seoul (South Korea), $\mathrm{n}=1000$ New York (NY, USA), $\mathrm{n}=766$ Manchester (UK), $\mathrm{n}=741$ Brompton (UK), $\mathrm{n}=689$ Belfast (UK), n=492 Guangzhou (China), n=389 Sweden, $n=290$ Leicester/Kings College Hospital (UK), and n=87 Northumbria (UK). Figure 1 illustrates the age and sex breakdown of patients presenting to all clinics with the Pie chart illustrating the sex ratio of the patients seen. Two-thirds of the patients $(n=6591,66 \%)$

Support statement: The authors gratefully acknowledge the support of the European Respiratory Society in funding the Task Force on Chronic Cough, which afforded the participants the opportunity to collaborate on this manuscript.

Conflict of interest: Disclosures can be found alongside the online version of this article at erj.ersjournals.com 

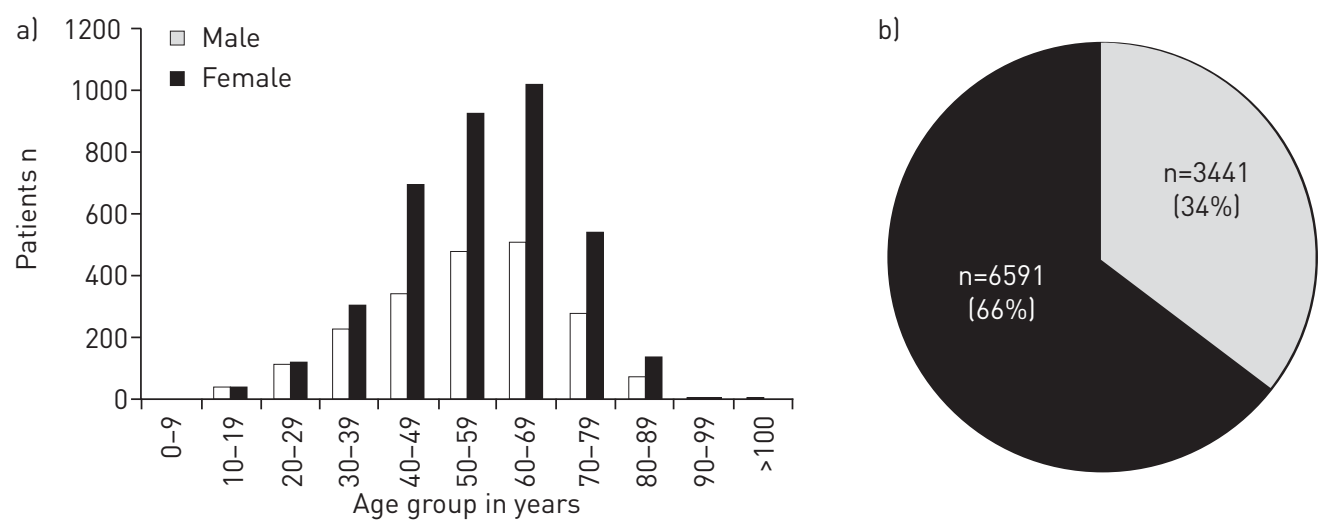

FIGURE 1 Age and sex distribution of all the patients presenting to the clinics.

attending the clinics were females. This female preponderance was consistent in each individual cough clinic although less prominent in some smaller clinics; Hull $(n=1373,62 \%)$, Holland $(n=1233,67 \%)$, Seoul $(n=1043,69 \%)$, New York $(n=698,70 \%)$, Manchester $(n=549,72 \%)$, Brompton $(n=508,69 \%)$, Belfast $(n=448,65 \%)$, Guangzhou $(n=251,51 \%)$, Sweden $(n=262,67 \%)$ Leicester/Kings College Hospital $(\mathrm{n}=177,61 \%)$, and Northumbria $(\mathrm{n}=49,56 \%)$ (table 1). Figure S1 illustrates the patient profile for each individual UK clinic.

The mean $\pm \mathrm{SD}$ age was $55 \pm 14.97$ years. Holland 57 years, Hull 57 years, Seoul 52 years, New York 58 years, Manchester 57 years, Brompton 55 years, Belfast 56 years, Guangzhou 42 years, Sweden 54 years, Leicester/Kings College Hospital 56 years, and Northumbria 58 years. The most common age for presentation was 60-69 years. This was also true among the individual clinics, with the exception of Guangzhou where 30-39 years was the most common for presentation. Chronic cough was most commonly

TABLE 1 Age and sex distribution of patients presenting to cough clinics within each country

\begin{tabular}{|c|c|c|c|c|c|c|c|c|c|c|c|c|}
\hline \multirow[t]{2}{*}{ Country } & \multirow[t]{2}{*}{ Totals } & \multicolumn{11}{|c|}{ Age group in years } \\
\hline & & $0-9$ & $10-19$ & $20-29$ & $30-39$ & $40-49$ & $50-59$ & $60-69$ & $70-79$ & $80-89$ & $90-99$ & $\geqslant 100$ \\
\hline \multicolumn{13}{|l|}{ UK $n=4792$} \\
\hline Total & & 0.02 & 1.29 & 2.80 & 7.35 & 17.3 & 24.7 & 28.7 & 14.7 & 3.00 & 0.08 & 0.02 \\
\hline Female & 65 & 100 & 53 & 55 & 61 & 68 & 65 & 64 & 65 & 67 & 75 & 0 \\
\hline Male & 35 & 0 & 47 & 45 & 39 & 32 & 35 & 36 & 35 & 33 & 25 & 100 \\
\hline \multicolumn{13}{|c|}{ USA $n=1000$} \\
\hline Total & & 0.00 & 0.40 & 3.00 & 7.40 & 17.20 & 23.9 & 23.2 & 16.6 & 7.40 & 0.90 & 0.00 \\
\hline Female & 70 & 0 & 50 & 57 & 59 & 70 & 69 & 75 & 74 & 64 & 89 & 0 \\
\hline Male & 30 & 0 & 50 & 43 & 41 & 30 & 31 & 25 & 26 & 36 & 11 & 0 \\
\hline \multicolumn{13}{|c|}{ Holland $n=1841$} \\
\hline Total & & 0.00 & 1.74 & 2.34 & 7.77 & 15.5 & 26.5 & 26.7 & 15.6 & 3.75 & 0.16 & 0.00 \\
\hline Females & 67 & 0 & 75 & 56 & 63 & 66 & 67 & 68 & 67 & 72 & 100 & 0 \\
\hline Males & 33 & 0 & 25 & 44 & 37 & 34 & 33 & 32 & 33 & 28 & 0 & 0 \\
\hline \multicolumn{13}{|c|}{ Sweden $n=389$} \\
\hline Total & & 0.00 & 1.29 & 9.77 & 8.48 & 15.9 & 20.8 & 29.3 & 12.6 & 1.80 & 0.00 & 0.00 \\
\hline Females & 67 & 0 & 60 & 79 & 45 & 69 & 62 & 73 & 65 & 86 & 0 & 0 \\
\hline Males & 33 & 0 & 40 & 21 & 55 & 31 & 38 & 27 & 35 & 14 & 0 & 0 \\
\hline \multicolumn{13}{|c|}{ South Korea $n=1518$} \\
\hline Total & & 0.07 & 2.44 & 9.35 & 12.5 & 14.6 & 22.6 & 25.5 & 11.9 & 1.05 & 0.07 & 0.00 \\
\hline Female & 69 & 0 & 35 & 47 & 63 & 76 & 75 & 74 & 67 & 44 & 100 & 0 \\
\hline Male & 31 & 100 & 65 & 53 & 37 & 24 & 25 & 26 & 33 & 56 & 0 & 0 \\
\hline \multicolumn{13}{|c|}{ China $n=492$} \\
\hline Total & & 0.20 & 2.64 & 16.46 & 27.9 & 24.6 & 14.8 & 8.94 & 3.86 & 0.61 & 0.00 & 0.00 \\
\hline Females & 51 & 100 & 46 & 38 & 49 & 50 & 74 & 52 & 42 & 33 & 0 & 0 \\
\hline Male & 49 & 0 & 54 & 62 & 51 & 50 & 26 & 48 & 58 & 67 & 0 & 0 \\
\hline
\end{tabular}

The data are presented as percentages. 
presented in older patients, with $6821(68 \%)$ of the patients aged $>50$ years, $4441(44 \%)$ aged $>60$ years, and $1783(18 \%)$ aged $\geqslant 70$ years.

10 males with a mean (interquartile range) age of 28.7 (19-47) years and 10 females aged 27.3 (21-33) years underwent capsaicin challenge. The maximum tolerable dose of inhaled capsaicin was significantly lower in females when compared to males (geometric mean \pm SEM was $2.2 \pm 1.3 \mu \mathrm{M}$ versus $7.2 \pm 1.4 \mu \mathrm{M}$, respectively, $\mathrm{p}<0.01$ ) (fig. 2). Capsaicin inhalation was associated with activations in central-cough neural networks as detected for by fMRI, including regional responses in the sensory, motor and limbic cortices (data not shown). The magnitude of the regional responses was significantly larger in the primary somatosensory cortices (SM1) of females compared to males (left SM1 p $<0.001$ and right SM1 p $<0.01$ ) (figs 3 and 4), despite the lower mean challenge dose of capsaicin used in females.

\section{Discussion}

The age and sex distribution in this large group of patients across three continents was strikingly uniform. The over representation of females in the patient population presenting with a primary complaint of chronic cough has been long recognised [7, 13], and is perhaps the most striking example of sex-related differences in respiratory pathophysiology. Whilst often mentioned in the discussion of papers concerned with cough, it is frequently ignored in reviews dealing with the structure and function of the male and female respiratory tract [14]. Epidemiological surveys reveal a preponderance of females complaining of a chronic cough in the unselected general population [15]. Twice as many females as males develop a cough on angiotensin-converting-enzyme inhibitor therapy [16]. Females have a heightened sensitivity of the cough reflex as revealed by inhalational challenge with citric acid [17, 18], tartaric acid [19], and capsaicin [20]. This increased sensitivity of the cough reflex is also demonstrable in patients attending the cough clinic [21] where objectively recorded cough in females is twice that of males [18]. Together these observations would suggest that females have an increased sensitivity of the cough reflex in comparison with males. Thus, for any given stimulus within a population, females would be expected to present with a greater frequency and in our survey the rate is twice that of males.

We, therefore, studied functional brain activity in response to capsaicin inhalation in healthy males and females in order to assess possible differences in the neural processing in coughing. We have previously shown that capsaicin inhalation activates a distributed neural network in the human brain in response to an urge-to-cough irritation. Females were more sensitive to capsaicin challenge and despite the lower stimulus the magnitude of the activation in the somatosensory cortex was approximately twice that of males. The region of the somatosensory cortex displaying the sex-related differences is known to receive airway sensory inputs and is activated in close correlation with an individual's perceived urge-to-cough intensity [10]. The larger somatosensory response in healthy females, despite the lower provoking stimulus, suggests a possible mechanism for observed sex-related distribution of patients presenting to cough clinics. However, we are unable to say whether our fMRI observations of sex-related differences in cough processing are applicable to patients with chronic cough, nor whether comparable age-dependent alterations in central processing of airway irritant stimuli occur. Although, a recent study has demonstrated that the technique is sensitive to interventions that are known to suppress cough [11]. Sex-related dimorphism, in the processing of other noxious stimuli, has been widely reported. Females typically display lower cutaneous and visceral pain thresholds than do males and are more likely to present clinically with many chronic pain conditions [22, 23]. Evidence from functional brain imaging studies indicates that this may also be due to differential responses in females and males [24].

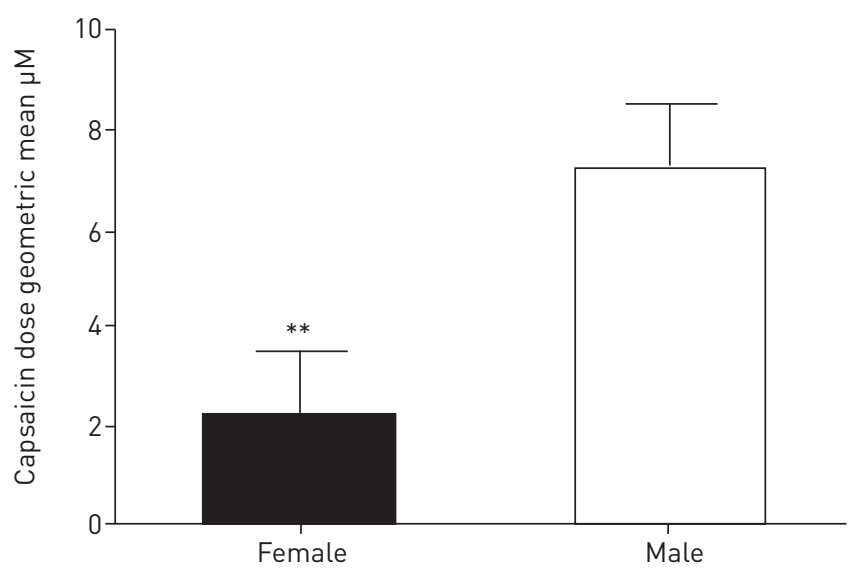

FIGURE 2 The maximum dose of capsaicin that could be inhaled without coughing was measured for a group of young, healthy females $(\mathrm{n}=10)$ and males $(\mathrm{n}=10)$. The geometric mean of the tolerable dose was significantly lower in females, the t-test with 18 degrees of freedom was 2.9. ${ }^{*}: \mathrm{p}<0.01$. 

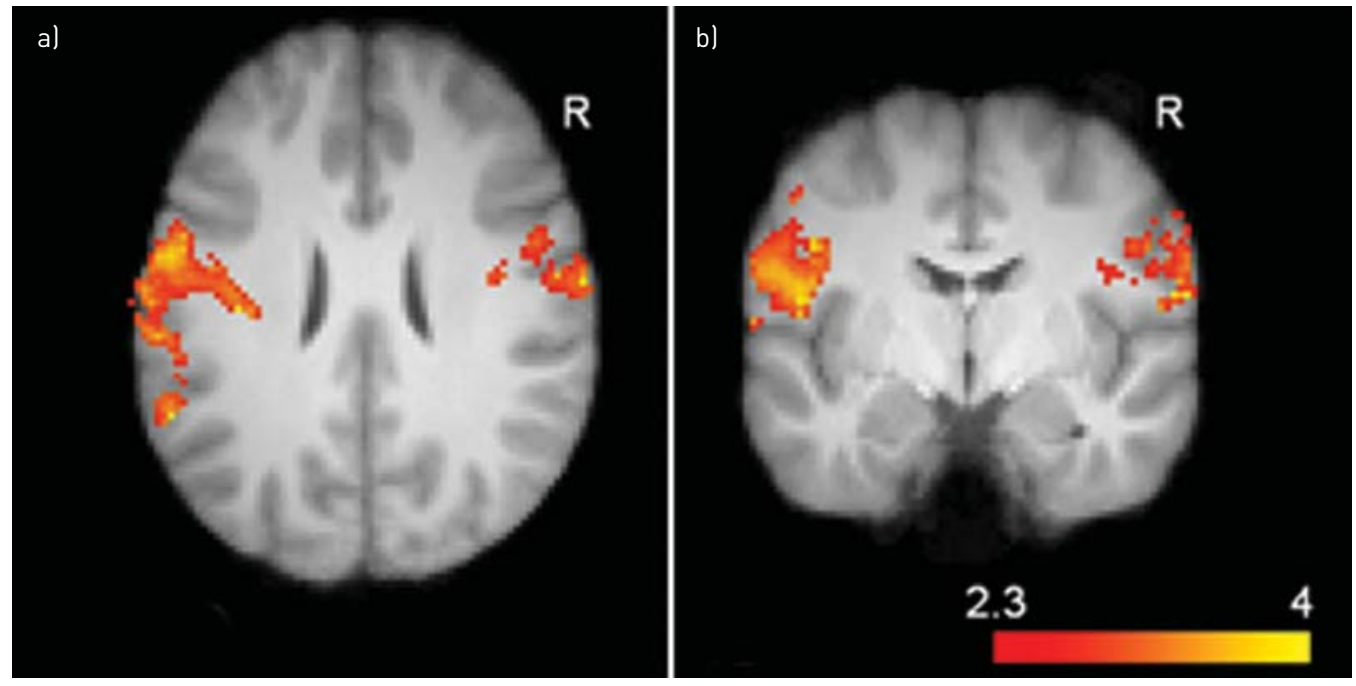

FIGURE 3 Regional brain responses in axial and coronal views during inhalation of the tolerable dose of capsaicin. These functional brain images show the location of the activations that were significantly different in young healthy females compared to males. a) The region of somatosensory cortex showing sex-related differences was located at the lower part of the central sulcus, seen here in axial view $26 \mathrm{~mm}$ above the anterior commissure. b) The lower part of the central sulcus ends above the lateral sulcus, which can be seen in this coronal slice, $6 \mathrm{~mm}$ behind the anterior commissure, as a darker horizontal line below the rendered activations in both hemispheres. This lower part of the somatosensory cortex receives afferent inputs from the airways.

There is a striking difference in the age distribution of patients attending cough clinics with a preponderance of middle aged or older subjects. Over two-thirds of the patients were aged $\geqslant 50$ years and a fifth were aged $\geqslant 70$ years. This is similar to the prevalence of many other chronic diseases including gastrooesophageal reflux [25]; a condition that is commonly associated with a chronic cough. However, it is unlike the demographic profile of atopic asthma, inferring that this may not be an important factor in disease pathogenesis in chronic cough.

Although the number of younger people seen in our clinics is limited, there appears to be a much more even distribution of the sexes. CHANG et al. [26] have studied the cough-reflex sensitivity of boys and girls and found there is no sex- related difference. It is, therefore, possible that the greater sensitivity of the cough reflex seen in females arises after puberty. VARECHOVA et al. [27] found that in contrast to younger children late pubertal girls had heightened cough sensitivity, which would be in keeping with the above hypothesis. We suggest heightened cough sensitivity in females of childbearing age may have evolutionary advantage by helping to prevent aspiration in pregnancy. A simple, direct effect of circulating hormones is unlikely to be responsible since we clearly demonstrate that the female preponderance continues well after the menopause. Indeed, the "typical" patient presenting to a cough clinic is a perimenopausal female. Hormone-induced anatomical changes in the female brain have been described in animal experiments [28].

FIGURE 4 Quantification of the size of the regional responses in the right and left primary somatosensory cortices (SM1) of females and males, as represented by mean parameter estimates. Activations were significantly greater in the females compared to the males in the left and the right hemispheres. ${ }^{* *}: \mathrm{p}<0.01$.

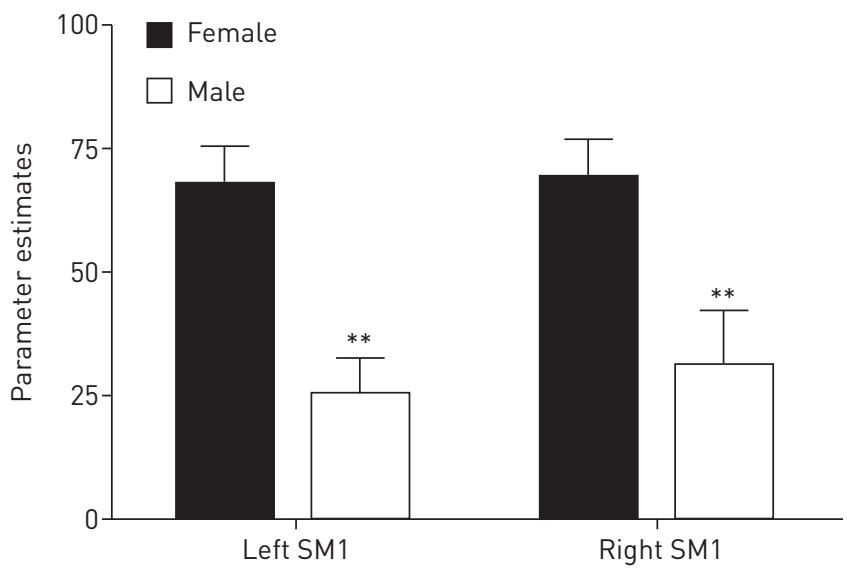


Our study has a number of limitations. As a retrospective survey contemporary verification in terms of diagnosis and investigations was not performed, although, since each clinic was a specialist centre adherence to international guidelines on the management of chronic cough would perhaps be expected. The lack of prospective uniform data collection also precludes the accurate estimation of other important variables such as smoking history, physiological variables and comorbidities. These elements will clearly need to be addressed in future investigations. In our survey the referral pattern to specialist cough clinics may not reflect the true epidemiology of chronic cough in the population. Thus our conclusion that there is a uniform clinical syndrome is based on patients who have cough refractory to first-line treatment by primary care physicians and may be unrepresentative of the general population. Such patients may have difficulties in access to clinics and thus to engage in a consultation. At the extreme of age, older patients may experience difficulty in obtaining and undertaking referral to a distant specialist clinic. A survey of patients responding to a BBC Radio 4 programme on chronic cough displayed an older patient profile possibly suggesting the demographic profile of the audience but also perhaps signifying unrecognised morbidity [29].

Similar factors may explain the outlying results from some of the smaller cough clinics particularly Guangzhou. These show a more equal sex distribution and presentation at a younger age in the Chinese population. Whilst there may be a genuine ethnic difference in cough-reflex sensitivity no differences were seen in a study of Chinese-American volunteers [30]. Environmental factors, such as air pollution, may be responsible. High levels of outdoor air pollution are positively associated with persistent cough in China [31, 32].

True assessment of the association between sex, aging and chronic cough awaits a cross-sectional analysis of unselected patients, but it is tempting to speculate that these demographic characteristics identify chronic cough as a distinct disease process.

In recent years a unifying diagnosis for patients with "idiopathic" chronic cough has become increasingly accepted [33]. The cough hypersensitivity syndrome is characterised by a heightened response of afferent nociceptors within the upper airways and presents with a distinctive clinical history of discrete precipitating triggers, associated upper-airway symptoms and atypical features of gastro-oesophageal reflux [34]. The concept of cough hypersensitivity syndrome is clearly endorsed by experts in this field, as suggested by the ERS task force report [5]. This may mimic or co-exist with other pulmonary or extra-pulmonary conditions, a view supported by the ERS task force. Specific questionnaires eliciting these associations reveal $>90 \%$ specificity for diagnosis in patients presenting to a specialist cough clinics. Our findings that there is essentially no difference between the patient profile across three continents and 11 clinics gives credence to the hypothesis that these patients have a single, common, clinical entity. We suggest that the cough hypersensitivity syndrome best explains the observed distribution within this patient population.

\section{Acknowledgements}

We acknowledge contributions from the following: O. Usmani, (Royal Brompton and Harefield NHS Trust and National Heart and Lung Institute, Imperial College, London, UK), J. Hull (Proctor and Gamble Ltd, Egham, UK), A. Rigby (Centre for Cardiovascular and Metabolic Research, Hull York Medical School, Cottingham, UK), C. Brightling (Respiratory Medicine, Glenfield Hospital, Leicester, UK) and A. Woodcock (Centre for Respiratory and Allergy, University of Manchester, University Hospital of South Manchester, Manchester, UK).

\section{References}

Cullinan P. Persistent cough and sputum: prevalence and clinical characteristics in south east England. Resp Med 1992; 86: 143-149.

2 Pratter MR, Brightling CE, Boulet LP, et al. An empiric integrative approach to the management of cough: ACCP evidence-based clinical practice guidelines. Chest 2006; 129: Suppl. 1, 222S-231S.

3 French CL, Irwin RS, Curley FJ, et al. Impact of chronic cough on quality of life. Arch Intern Med 1998; 158 : $1657-1661$.

4 Dicpinigaitis PV, Tso R, Banauch G. Prevalence of depressive symptoms among patients with chronic cough. Chest 2006; 130: 1839-1843.

5 Morice AH, Millqvist E, Belvisi MG, et al. Expert opinion on the cough hypersensitivity syndrome in respiratory medicine. Eur Respir J 2014; 44; 1132-1148.

6 Morice AH, McGarvey L, Pavord I. Recommendations for the management of cough in adults. Thorax 2006; 61: Suppl. 1, i1-i24.

7 Morice AH. Epidemiology of cough. Pulm Pharmacol Ther 2002; 15: 253-259.

8 Johansson A, Millqvist E, Nordin S, et al. Relationship between self-reported odor intolerance and sensitivity to inhaled capsaicin: proposed definition of airway sensory hyperreactivity and estimation of its prevalence. Chest 2006; 129: 1623-1628.

9 Mazzone SB, McLennan L, McGovern AE, et al. Representation of capsaicin-evoked urge-to-cough in the human brain using functional magnetic resonance imaging. Am J Respir Crit Care Med 2007; 176: 327-332.

10 Farrell MJ, Cole LJ, Chiapoco D, et al. Neural correlates coding stimulus level and perception of capsaicin-evoked urge-to-cough in humans. Neuroimage 2012; 61: 1324-1335.

11 Leech J, Mazzone SB, Farrell MJ. Brain activity associated with placebo suppression of the urge-to-cough in humans. Am J Respir Crit Care Med 2013; 188: 1069-1075. 
12 Worsley KJ, Evans AC, Marrett S, et al. A three-dimensional statistical analysis for CBF activation studies in human brain. J Cereb Blood Flow Metab 1992; 12: 900-918.

13 Bende M, Millqvist E. Prevalence of chronic cough in relation to upper and lower airway symptoms; the Skövde population-based study. Front Physiol 2012; 3: 251.

14 Becklake MR, Kauffmann F. Gender differences in airway behaviour over the human life span. Thorax 1999; 54: 1119-1138.

15 Janson C, Chinn S, Jarvis D, et al. Determinants of cough in young adults participating in the European Community Respiratory Health Survey. Eur Respir J 2001; 18: 647-654.

16 Yeo WW, Foster G, Ramsay LE. Prevalence of persistent cough during long-term enalapril treatment: controlled study versus nifedipine. Q J Med 1991; 80: 763-770.

17 Rostami-Hodjegan A, Abdul-Manap R, Wright CE, et al. The placebo response to citric acid-induced cough: pharmacodynamics and gender differences. Pulm Pharmacol Ther 2001; 14: 315-319.

18 Kelsall A, Decalmer S, McGuinness K, et al. Sex differences and predictors of objective cough frequency in chronic cough. Thorax 2009; 64: 393-398.

19 Fujimura M, Sakamoto S, Kamio Y, et al. Sex difference in the inhaled tartaric acid cough threshold in non-atopic healthy subjects. Thorax 1990; 45: 633-634.

20 Dicpinigaitis PV, Rauf K. The influence of gender on cough reflex sensitivity. Chest 1998; 113: 1319-1321.

21 Kastelik JA, Thompson RH, Aziz I, et al. Sex related differences in cough reflex sensitivity in patients with chronic cough. Am J Respir Crit Care Med 2002; 166: 961-964.

22 Fillingim RB, King CD, Ribeiro-Dasilva MC, et al. Sex, gender, and pain: a review of recent clinical and experimental findings. J Pain 2009; 10: 447-485.

23 Racine M, Tousignant-Laflamme Y, Kloda LA, et al. A systematic literature review of 10 years of research on sex/ gender and experimental pain perception - part 1: are there really differences between women and men? Pain 2012; 153: 602-618.

24 Henderson LA, Gandevia SC, Macefield VG. Gender differences in brain activity evoked by muscle and cutaneous pain: a retrospective study of single-trial fMRI data. Neuroimage 2008; 39: 1867-1876.

25 Ness-Jensen E, Lindam A, Lagergren J, et al. Changes in prevalence, incidence and spontaneous loss of gastrooesophageal reflux symptoms: a prospective population-based cohort study, the HUNT study. Gut 2012; 61: 1390-1397.

26 Chang AB, Phelan PD, Sawyer SM, et al. Cough sensitivity in children with asthma, recurrent cough, and cystic fibrosis. Arch Dis Child 1997; 77: 331-334.

27 Varechova S, Plevkova J, Hanacek J, et al. Role of gender and pubertal stage on cough sensitivity in childhood and adolescence. J Physiol Pharmacol 2008; 59: Suppl. 6, 719-726.

28 Abel JL, Rissman EF. Location, location, location: genetic regulation of neural sex differences. Rev Endocr Metab Disord 2012; 13: 151-161.

29 Everett CF, Kastelik JA, Thompson RH, et al. Chronic persistent cough in the community: a questionnaire survey. Cough 2007; 3: 5.

30 Dicpinigaitis PV, Allusson VRC, Baldanti A, et al. Ethnic and gender differences in cough reflex sensitivity. Respiration 2001; 68: 480-482.

31 Pan G, Zhang S, Feng Y, et al. Air pollution and children's respiratory symptoms in six cities of Northern China. Respir Med 2010; 104: 1903-1911.

32 Lai HK, Ho SY, Wong CM, et al. Exposure to particulate air pollution at different living locations and respiratory symptoms in Hong Kong -an application of satellite information. Int J Environ Health Res 2010; 20: $219-230$.

33 McGarvey LP. Idiopathic chronic cough: a real disease or a failure of diagnosis? Cough 2005; 1: 9.

34 Morice AH, Faruqi S, Wright CE, et al. Cough hypersensitivity syndrome: a distinct clinical entity. Lung 2011; 189: 73-79. 